\title{
Pacific
}

Journal of

Mathematics

\section{THE EULER CLASS FOR “PIECEWISE” GROUPS}

\author{
Peter Abraham Greenberg
}




\section{THE EULER CLASS FOR “PIECEWISE” GROUPS}

\section{Peter Greenberg}

The Euler class is a semiconjugacy invariant of a discrete group $G$ of orientation preserving homeomorphisms of the circle. An element of the second cohomology group of $G$ with integral coefficients, it is often difficult to calculate, but even its nonvanishing seems related to dynamical complexity of $G$. In this note, we consider a family of discrete groups $\Gamma_{H, S}(p, q)$ of homeomorphisms of the circle, whose definition generalizes that of piecewise linear homeomorphisms. We define an invariant with which one can verify the vanishing of the Euler class in a surprising range of cases. On the other hand, the vanishing of the invariant, together with a simple geometric condition, assures the nonvanishing of the Euler class.

The invariant has a simple "operational" definition, but can also be interpreted as an element of the fundamental group of the classifying space of a certain pseudogroup. We also apply it to the question of the existence of elements of finite order in the groups $\Gamma_{H, S}(p, q)$.

This work was mostly completed while I was visiting the University of Geneva, whose support is gratefully acknowledged. I especially thank André Haefliger and Ana Maria Fereira da Silva for kindly commenting on early versions.

\section{Definitions and results.}

1.1. The groups. Let $H$ be a group of analytic, orientation preserving homeomorphisms of the real line $\mathbf{R}$, and let $S$ be an $H$-invariant subset of $\mathbf{R}$. Let $p<q$ be elements of $S$ in the same $H$-orbit. Let $S_{p, q}^{1}$ denote the closed interval $[p, q]$ with $p$ and $q$ identified as the basepoint. Define $\Gamma_{H, S}(p, q)$ to be the group of homeomorphisms $g$ of $S_{p, q}^{1}$ such that there exist $s_{i} \in S, p=s_{0}<\cdots<s_{n}=q$, so that the restriction of $g$ to $\left[s_{i}, s_{i+1}\right]$ agrees pointwise with an element $h_{i}$ of $H$. Thus, $\Gamma_{H, S}(p, q)$ is "the group of piecewise- $H$ homeomorphisms of $S_{p, q}^{1}$, with breakpoints in $S . "$

1.2. The invariant. Let $N=N(S)$ denote the normal subgroup of $H$ generated by all elements which fix some point in $S$. If $p, q \in S$ are in the same $H$-orbit, the equivalence class in $H / N$ of an element 
$h \in H$ such that $h(p)=q$ depends on $p$ and $q$ only. This equivalence class will be called the manifold class $h$ of the circle $S_{p, q}^{1}$.

1.3. A flexibility condition. We say that a pair $(H, S)$ is flexible if the following condition is satisfied: let $a, b, c, d \in S, a<b$, $c<d$. Suppose there are $g, h \in H$, with $g \equiv h$ in $H / N$, such that $g(a)=c, h(b)=d$. Then there are $s_{i} \in S, a=s_{0}<\cdots<s_{n}=b$, and $g_{i} \in H$ so that $g_{1}(a)=c, g_{n}(b)=d$, and $g_{i}\left(s_{i}\right)=g_{i+1}\left(s_{i}\right)$, $1 \leq i \leq n-1$. That is, there is a piecewise- $H$ homeomorphism from $[a, b]$ to $[c, d]$.

We can now state the main results, staying with the notation established above. Suppose that $G$ is a group acting on the circle. By the rational Euler class of $G$ we mean the rational reduction in $H^{2}(G ; \mathbf{Q})$ of the (integral) Euler class.

1.4. TheOREM. If no nonzero power of the manifold class of $S_{p, q}^{1}$ can be written as a product of commutators in $H / N$, then the rational Euler class of $\Gamma_{H, S}(p, q)$ vanishes.

One can restate the hypothesis; it requires that the manifold class be of infinite order in the abelianization of $H / N$.

1.5. Corollary. If $H / N$ is abelian and the manifold class is of infinite order, then the rational Euler class of $\Gamma_{H, S}(p, q)$ vanishes.

A partial converse to 1.4 is the following.

1.6. Theorem. Suppose that $(H, S)$ is flexible, and that the manifold class of $S_{p, q}^{1}$ is null. Then the Euler class is nonzero, and not divisible in $H^{2}\left(\Gamma_{H, S}(p, q) ; \mathbf{Z}\right)$.

Regarding elements of torsion, we have the following result.

1.7. TheOREM. Suppose there is a $g \in \Gamma_{H, S}(p, q)$ and $s \in[p, q] \cap$ $S$ such that the orbit $\left\{g^{k}(s)\right\}_{k \in \mathbf{Z}}$ has $m$ elements, $m<\infty$. Then the manifold class of $S_{p, q}^{1}$ is an mth power in $H / N$.

1.8. Corollary. If the manifold class is not divisible in $H / N$, $\Gamma_{H, S}(p, q)$ is torsion free.

Before stating results about divisibility of the integral Euler class and embeddings of surface groups, let us pause to consider two families of examples. 
1.9. Piecewise linear examples. Let $A$ be an additive subgroup of the real numbers, and let $U$ be a multiplicative subgroup of the positive real numbers, such that for all $a \in A, u \in U$, we have $u a \in A$. Let $H$ be the group of affine transformations of the form $h(x)=u x+a, u \in U, a \in A$, and let $S$ be the $H$-orbit of 0 , that is $A$. From ([Gr1], 1.13) we extract:

1.10. Proposition. (a) $(H, S)$ is flexible.

(b) $H / N \cong A /(u-1)$, the quotient of $A$ by the subgroup generated by all $u a-a, u \in U, a \in A$. If $h \in H, h(x)=u x+a$, the equivalence class of $h$ in $H / N$ is that of $a$ in $A /(u-1)$.

For example, take $r \in \mathbf{R}, r>1$, and let $A=Q\left[r, r^{-1}\right]$ be the ring of finite rational Laurent series in $r$. Let $U=\left\{r^{n}, n \in \mathbf{Z}\right\}$. Let $p=0, q=1$, and consider $\Gamma_{H, A}(0,1)$. The manifold class of $S_{0,1}^{1}$ is the equivalence class of $h(x)=x+1$ in $A /\left(r^{n}-1\right)$. This class is 0 if $r$ is algebraic and of infinite order if $r$ is transcendental, and the Euler class of $\Gamma_{H, A}(0,1)$ is nonzero or zero accordingly.

Note that the Euler class of $\Gamma_{H, A}(0, r-1)$ is always nonzero.

1.11. Piecewise projective examples. We begin with a slight modification of the original construction. Let $G$ be a group of orientation preserving analytic homeomorphisms of the circle $S^{1}$. Let $H=\widetilde{G}$ be the group of homeomorphisms of the universal cover $\widetilde{S}^{1}$ which cover elements of $G$. Recall that $H$ is a central extension $\mathbf{Z} \rightarrow H \rightarrow G$, where the $\mathbf{Z}=\pi_{1} S^{1}$ is identified as the group of covering transformations of $\widetilde{S}^{1}$ over $S^{1}$. Let $T$ denote the positive generator of $\pi_{1} S^{1}$ (that is $T x>x, x \in \widetilde{S}^{1}$ ).

Let $S$ be a $G$-invariant subset of $S^{1}$, and $\widetilde{S} \subseteq \widetilde{S}^{1}$ its inverse image. The manifold class of $S_{p, T_{p}}^{1}, p \in \widetilde{S}$, is simply $T \in H / N$; we consider the group $\Gamma_{H, \widetilde{S}}\left(p, T_{p}\right)$.

In particular we will consider subgroups $G$ of $\operatorname{PSL}_{2} \mathbf{R}$, acting as usual on $S^{1}=\mathbf{R} \cup\{\infty\}$ by linear fractional transformations. For example, take $G=\mathrm{PSL}_{2} F, F$ a subfield of $\mathbf{R}$, and let $S=F \cup\{\infty\}$. The results of [Gr2] imply:

1.12. Proposition. (a) $\operatorname{PSL}_{2}(F, \widetilde{S})$ is flexible.

(b) $\mathrm{PSL}_{2} F / N$ is the group with one element.

As a consequence, the Euler class of $\Gamma_{H, \widetilde{S}}\left(p, T_{p}\right)$ is nonvanishing. 
Now set $G=\mathrm{PSL}_{2} \mathbf{Z}$, and $S=\mathbf{Q} \cup\{\infty\}$ the $G$-orbit of 0 . The group $\Gamma_{H, \widetilde{S}}\left(p, T_{p}\right)$ can be shown (following a remark of Thurston) to be isomorphic to the "remarkable group" studied by Etienne Ghys and Vlad Sergiescu in [GhS]. It turns out ([GhS]) that $H, \widetilde{S}$ is flexible, and that $\Gamma_{H, \widetilde{S}}\left(p, T_{p}\right)$ has a nonzero Euler class.

The situation changes when we pass to congruence subgroups of $\mathrm{PSL}_{2} \mathbf{Z}$. Consider $G=\Gamma(M)$, the set of matrices congruent to $\pm I$ modulo $M \in \mathbf{Z}, M>1$. It is known (see [Sh], Chap. 1) that $\Gamma(M)$ is a free group. More precisely, a model for $K(\Gamma(M), 1)$ is a certain Riemann surface with punctures; $\mathbf{Q} \cup\{\infty\}$ breaks up into a finite number of $\Gamma(M)$-orbits, corresponding to the punctures. A small circle about a puncture is the conjugacy class in $\Gamma(M)$ of an element fixing a point in the corresponding orbit. If the surface has nonzero genus, the group is not generated by these stabilizers.

Let $S$ be the union of one or more of these orbits. Since any central extension of a free group is trivial, $H \cong G \times \mathbf{Z}$, and $T$ corresponds to $(e, 1)$; further, $H / N \cong G / N \times \mathbf{Z}$, where $N$ denotes the subgroup of $G$ generated by stabilizers of points in $S$. Thus if the genus of $G$ is nonzero, the Euler class of $\Gamma_{H, \widetilde{S}}\left(p, T_{p}\right)$ is 0 . Or, if the genus of $G$ is zero, but $S$ does not contain at least two of the orbits which comprise $\mathbf{Q} \cup\{\infty\}$, the Euler class of $\Gamma_{H, \widetilde{S}}\left(p, T_{p}\right)$ vanishes.

1.13. Divisibility and surface groups. The following rather technical result will be combined with the Milnor-Wood inequality ([Mi], [W]) to obtain a result (1.15) on embedding surface groups in the $\Gamma_{H, S}(p, q)$.

1.14. Proposition. Suppose that $H / N$ is abelian, that the manifold class of $S_{p, q}^{1}$ is nonzero, and that the rational Euler class of $\Gamma_{H, S}(p, q)$ is nonzero. Then the manifold class of $S_{p, q}^{1}$ is torsion, and its order in $H / N$ divides the integral Euler class.

Let $\pi_{g}=\left\langle a_{i}, b_{i}, i=1, \ldots, g \mid \pi\left[a_{i}, b_{i}\right]=1\right\rangle$ be the fundamental group of a compact surface of genus $g$. Etienne Ghys has asked ([Gh3]) for conditions under which $\pi_{g}$ may be embedded into certain subgroups of homeomorphisms of the circle.

1.15. Corollary. Suppose that $H / N$ is abelian, that the manifold class of $S_{p, q}^{1}$ is nonzero, and that there is a homeomorphism $f: \pi_{g} \rightarrow$ $\Gamma_{H, S}(p, q)$ such that the pullback to $H^{2}\left(\pi_{g} ; \mathbf{Z}\right)$ of the Euler class is nonzero. 
Then the manifold class is torsion, and its order divides a natural number less than $2 g-1$.

Proof. By the assumptions and 1.14, the manifold class is torsion, and its order divides the Euler class of $\Gamma_{H, S}(p, q)$, and hence its order divides the pullback of $E$ in $H^{2}\left(\pi_{g} ; \mathbf{Z}\right)$. But the Milnor-Wood inequality asserts that this pullback is at most $\pm(2 g-2)$ times the generator of $H^{2}\left(\pi_{g} ; \mathbf{Z}\right)$. Consequently, the order of the manifold class divides some natural number less than $2 g-1$.

1.16. ExAmple. Consider the piecewise linear example (1.10) with $A=\mathbf{Z}\left[\frac{1}{n}\right], n \in \mathbf{Z}, n>1$, and $U=\left\{n^{k}\right\}_{k \in \mathbf{Z}}$. Then $H / N \cong$ $\mathbf{Z} / n-1$, and the manifold class of $S_{0,1}^{1}$ generates. Consequently, for a homomorphism $\pi_{t} \rightarrow \Gamma_{H, S}\left(S_{0,1}^{1}\right)$ to pull back a nonzero Euler class, it is necessary that $n-1$ divide one of $2, \ldots, 2 g-2$. In particular, $n \leq 2 g$.

1.17. Organization. In the next section we give proofs of the results above, save 1.6. In $\S 3$ we interpret the manifold class as an element of the fundamental group of a certain pseudogroup, and prove 1.6.

2. The homomorphism $C$. After introducing some notation, we enunciate a Lemma 2.1 and use it to prove various results. Then the lemma is proved. We continue with the notation already established, and consider an $H, S$ and $p, q \in S, p<q$.

Let $\widetilde{S}^{1}$ denote the universal cover of $S_{p, q}^{1}$, and let $T$ denote the positive generator of the group of covering transformations of $S_{p, q}^{1}$. Let $\widetilde{\Gamma}_{H, S}(p, q)$ denote the group of lifts of elements of $\Gamma_{H, S}(p, q)$ to homeomorphisms of $\widetilde{S}^{1}$. Choose a lift $\tilde{p}$ of $p$. Let $\widetilde{S}$ denote the set of lifts of points in $S$ to $\widetilde{S}^{1}$.

2.1. Lemma. There is a homomorphism $C: \widetilde{\Gamma}_{H, S} \rightarrow H / N$ such that

(i) $C(T)$ is the manifold class of $S_{p, q}^{1}$.

(ii) $C\left(g_{1}\right)=C\left(g_{2}\right)$ if $g_{1}(s)=g_{2}(s), s \in \widetilde{S}$.

Proof of 1.4. Suppose that the rational Euler class of $\Gamma_{H, S}(p, q)$ is nonzero. Then there are $a_{i}, b_{i} \in \Gamma_{H, S}(p, q), i=1, \ldots, k$, such that, choosing lifts $\tilde{a}_{i}, \tilde{b}_{i} \in \widetilde{\Gamma}_{H, S}(p, q)$, we have $\prod_{i=1}^{k}\left[\tilde{a}_{i}, \tilde{b}_{i}\right]=T^{m}$, $m \neq 0$. Applying the homomorphism $C$ of 2.1 , we see that the $m$ th power of the manifold class is a product of commutators. 
Proof of 1.7. Suppose that $g \in \Gamma_{H, S}(p, q)$, and that for $s \in S \cap$ $[p, q],\left\{g^{k}(s)\right\}_{k \in \mathbf{Z}}$ has cardinality $m<\infty$. Possibly replacing $g$ with a power of $g$, there is a lift $\tilde{g} \in \widetilde{\Gamma}_{H, S}(p, q)$, and a lift $\tilde{s} \in S$, such that $\tilde{g}^{m}(\tilde{s})=T \tilde{s}$. Applying 2.1 (ii), $C(\tilde{g})^{m}$ is the manifold class of $S_{p, q}^{1}$.

Proof of 1.14. Since the rational Euler class is nonzero, the integral Euler class is nonzero, and further, not torsion. Consequently, there is some least $m \neq 0$ such that the $m$ th power of the manifold class is a product of commutators in $H / N$. The latter being abelian, this $m$ th power is zero. Thus, viewed as a homomorphism from $\mathrm{H}_{2}\left(\Gamma_{H, S}(p, q) ; \mathbf{Z}\right)$ to $\mathbf{Z}$, the image of the Euler class is contained in $m \mathbf{Z}$. Thus, the Euler class is divisible by $m$.

We now construct the homomorphism $C$, and prove Lemma 2.1. As it happens, the natural domain of $C$ is a certain collection of homeomorphisms between open subsets of the line. Namely, let $g: U \rightarrow V$ be a homeomorphism between open subsets of the line. Then $g \in$ $\Gamma_{H, S}$, and we say $g$ is piecewise- $H$ with breakpoints in $S$ if there exists a subset $X$ of $U \cap S, X$ discrete in $U$, such that for any connected component $K$ of $U-X$, the restriction of $g$ to $K$ agrees pointwise with an element $h_{K}$ of $H$. We call $X$ the set of breakpoints of $g$. The set of homeomorphisms $\Gamma_{H, S}$ is a pseudogroup ([Ha]); it is closed under restriction to open subsets, taking inverses, and (where defined) composition.

2.2. Lemma. Let $g \in \Gamma_{H, S}$ with connected domain $U$. Let $X$ be the set of breakpoints of $g$, and let $h_{K} \in H$ denote the restriction of $g$ to a component $K$ of $U-X$. Then the equivalence class of $h_{K}$ in $H / N$ is independent of the component $K$.

Proof. We must show that if $K, K^{\prime}$ are two components of $U-X$, then $h_{K}=h_{K^{\prime}}$ in $H / N$. Since $X$ is discrete in $U$, we can assume that $K$ and $K^{\prime}$ are adjacent, that is $\bar{K} \cap \overline{K^{\prime}}=s \in S$. Then $h_{K}(s)=h_{K^{\prime}}(s)$, since $g$ is continuous. Thus $h_{K} h_{K^{\prime}}^{-1}(s)=s$, so that $h_{K} h_{K^{\prime}}^{-1} \in N$.

2.3. Definition. Let $g \in \Gamma_{H, S}$, with connected domain. We denote by $\sigma(g)$ the common value of the $h_{K}$ in $H / N$.

2.4. Lemma. Let $g_{i}: U_{i} \rightarrow V_{i}, i=1,2, g_{i} \in \Gamma_{H, S}$, with $U_{i}$ connected. If $V_{1} \subseteq U_{2}$, then $\sigma\left(g_{2} \circ g_{1}\right)=\sigma\left(g_{2}\right) \sigma\left(g_{1}\right)$.

Proof. Left to the reader. 
2.5. Lemma. Let $g_{1}, g_{2} \in \Gamma_{H, S}$ with connected domains whose intersection contains a point $s \in S$. If $g_{1}(s)=g_{2}(s)$, then $\sigma\left(g_{1}\right)=$ $\sigma\left(g_{2}\right)$.

Proof. Restricting to subsets if necessary, we can assume that $g_{1}$ and $g_{2}$ have a common range $V$ which contains $g_{1}(s)=g_{2}(s)$. Thus $g_{1}^{-1} g_{2}$ is well-defined, and $g_{1}^{-1} g_{2}(s)=s$. Consequently, the restriction of $g_{1}^{-1} g_{2}$ to a small interval to the right of $s$ agrees with an element of $N$, and so $\sigma\left(g_{1}\right)=\sigma\left(g_{2}\right)$.

Proof of 2.1. Pick $h \in H$ so that $h(p)=q$. Let $U=\bigcup_{n \in \mathbf{Z}} h^{n}[p, q]$. Then $U$ is a connected open subset of $\mathbf{R}$ on which the infinite cyclic group $\left\{h^{k}\right\}_{k \in \mathbf{Z}}$ acts, properly discontinuously.

Choose a lift $L:[p, q] \rightarrow \widetilde{S}^{1}$, that is, a continuous map such that the diagram

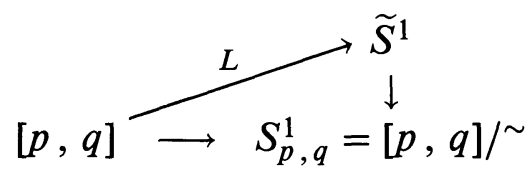

commutes, and such that $L(p)=\tilde{p}$. There is a unique homeomorphism $f: \widetilde{S}^{1} \rightarrow U$ such that $f L(x)=x, x \in[p, q]$, and such that $f \circ T=h \circ f$. Indeed, every $x \in \widetilde{S}^{1}$ can be written uniquely as $T^{k} L(y)$, for some $y \in[p, q)$; we define $f(x)=h^{k}(y)$.

It now follows that if $g \in \widetilde{\Gamma}_{H, S}(p, q)$ then $f g f^{-1} \in \Gamma_{H, S}$. Define $C(g)=\sigma\left(f g f^{-1}\right)$. Then $C(T)=\sigma\left(f T f^{-1}\right)=h$, and 2.5 implies that $C(g)$ depends only on $g(s), s \in \widetilde{S}$.

3. Haefliger structures on the circle. In this section we describe a general context for the results of this paper. We use the language of pseudogroups and their classifying spaces as developed by André Haefliger ([Ha]). A result of Dusa McDuff, ([McD]) as presented by Etienne Ghys and Vlad Sergiescu ([GhS]) is applied to prove 1.6.

Let $\Gamma$ be a pseudogroup of orientation preserving homeomorphisms between open subsets of the line. We shall assume:

3.1. Assumption. Every germ in $\Gamma$ admits an extension to a homeomorphism of the real line which is in $\Gamma$.

We can think of $\Gamma$ as its space of germs, with the sheaf topology. Then 3.1 implies that $\pi_{0} \Gamma$ inherits the structure of a group from $\Gamma$. The construction of the homomorphism $C$ in $\S 2$ proves: 
3.2. Proposition. $\pi_{0} \Gamma_{H, S} \cong H / N$. Indeed, the function $\sigma$ of 2.3 gives the isomorphism.

Associated to a pseudogroup $\Gamma$ is its classifying space $B \Gamma$, and it follows from Assumption 3.1 that $\pi_{1} B \Gamma \cong \pi_{0} \Gamma$. Now, $\pi_{1} B \Gamma$ is identified in the theory of Haefliger ([Ha]) with the set of homotopy classes of $\Gamma$-structures on $S^{1}$ with a distinguished basepoint. Indeed, given $\underline{h} \in \pi_{0} \Gamma$ represented by $h \in \Gamma$, such that for some $p \in \mathbf{R}, q=$ $h(p)>p$, we can construct a $\Gamma$-structure on $S^{1}$ corresponding to $\underline{h} \in$ $\pi_{0} \Gamma=\pi_{1} B \Gamma$ by "gluing $p$ to $q$ using $h$ " as described below. Hence, what we defined in 1.2 as the manifold class of $S_{p, q}^{1}$ corresponds, via 3.2 , to the element of $\pi_{1} B \Gamma$ which $S_{p, q}^{1}$, with basepoint $p \sim q$ and its $\Gamma_{H, S}$-structure, represents.

We now make precise the "gluing" construction referred to above. Pick a global extension of $h$ in $\Gamma$, to a homeomorphism of $\mathbf{R}$ which we will also denote $h \in \Gamma$ (using 3.1). Let $U=\bigcup_{n \in \mathbf{Z}} h^{n}[p, q]$. This is a connected open interval in $\mathbf{R}$, on which the infinite cyclic group $C_{n}=\left\{h^{k}\right\}_{k \in \mathbf{Z}}$ acts properly discontinuously. We define $S_{h}^{1}$ as $U$ modulo the action of $C_{h}$. Then $S_{h}^{1}$, with basepoint $p$, is a circle with $\Gamma$-structure corresponding to $\underline{h} \in \pi_{0} \Gamma$.

Let $\left.\Gamma\right|_{U}$ denote the pseudogroup $\Gamma$ restricted to $U$. The group $C_{h}$ acts on the space $\left.\Gamma\right|_{U}$ on the left and the right, by composition, and the quotient, denoted $\Gamma_{h}$, is the sheaf of locally- $\Gamma$ homeomorphisms between open subsets of $S_{h}^{1}$.

Let $\Gamma\left(S_{h}^{1}\right)$ be the group of global sections of $\Gamma_{h}$ over $S_{h}^{1}$, such that composition with the target map gives a homeomorphism of $S_{h}^{1}$. Then $\Gamma\left(S_{h}^{1}\right)$ is the group of locally- $\Gamma$ homeomorphisms of $S_{h}^{1}$. When $\Gamma=\Gamma_{H, S}, \Gamma\left(S_{h}^{1}\right)$ is precisely the group $\Gamma_{H, S}(p, q)$. We generalize 1.4 as follows.

3.3. Proposition. If no nonzero power of $\underline{h}$ is a commutator in $\pi_{0} \Gamma$, then the rational Euler class of $\Gamma\left(S_{h}^{1}\right)$ vanishes.

Proof (Sketch). As in $\S 2$, we must define a homomorphism $C: \widetilde{\Gamma}\left(S_{h}^{1}\right)$ $\rightarrow \pi_{0} \Gamma$, where $\widetilde{\Gamma}\left(S_{h}^{1}\right)$ is the group of homeomorphisms of $\widetilde{S}_{h}^{1}$ which cover elements of $\Gamma\left(S_{h}^{1}\right)$. Having constructed $S_{h}^{1}$ as a quotient of $U=\widetilde{S}^{1}$, we see that $\widetilde{\Gamma}\left(S_{h}^{1}\right)$ is the subgroup of the group $\Gamma(U)$ of $\Gamma$ homeomorphisms of $U$, consisting of elements which commute with $h$. Then $C$ is just the composition $\widetilde{\Gamma}\left(S_{h}^{1}\right) \rightarrow \Gamma(U) \rightarrow \pi_{0} \Gamma$. The rest of the proof is as in $\S 2$. 
In order to prove 1.6, we will invoke a theorem of Dusa McDuff [McD] to provide a homological model of the Eilenberg-Mac Lane spaces $B \Gamma_{H, S}(p, q)$, when $(H, S)$ is flexible. We begin by recalling some properties of the free loop space.

3.4. Remarks on $L X$. Suppose that $X$ is a connected and simply connected space. Let $\Lambda X$ denote the space of maps from $S^{1}$ to $X$, without regard to basepoint. The group $\mathrm{Homeo}^{+} S^{1}$ of orientation preserving homeomorphisms of $S^{1}$ acts on $\Lambda X$ on the left (composition with the inverse) and we denote by $L X$ the homotopy quotient $\left(E\right.$ Homeo $\left.^{+} S^{1}\right) \times_{\text {Homeo }+S^{1}}(\Lambda X)$. Then $L X$ is functorial in $X$. The evident map $X \rightarrow *$ induces a map $L X \rightarrow L^{*}=B \mathrm{Homeo}^{+} S^{1}$, and thus we can pull the Euler class of $B \mathrm{Homeo}^{+} S^{1}$ back to an element of $H^{2}(L X ; \mathbf{Z})$, which we call the Euler class of $L X$.

3.5. LEMMA. If $X$ is connected and simply connected, the Euler class of $L X$ is nonzero and not divisible.

Proof. Apply the construction $L$ to the inclusion and retraction of a point in $X$. We obtain $B \mathrm{Homeo}^{+} S^{1} \rightarrow L X \rightarrow B \mathrm{Homeo}^{+} S^{1}$ whose composition is homotopic to the identity, whence the lemma.

3.6. Germ-connectedness and flexibility. In [McD] Dusa McDuff proved that there is a map $f: B \operatorname{Diff}^{+} S^{1} \rightarrow L B \Gamma^{\infty}$, where $\operatorname{Diff}^{+} S^{1}$ is the discrete group of orientation preserving diffeomorphisms of the circle and $\Gamma^{\infty}$ is the pseudogroup of orientation preserving diffeomorphisms between open subsets of $\mathbf{R}$, such that $f$ induces an isomorphism in homology, and further, such that the pullback by $f$ of the Euler class of $L B \Gamma^{\infty}$ is the Euler class of $\operatorname{Diff}^{+} S^{1}$. (The latter from the construction of $f$ as a quotient of the map between certain circle bundles). It turns out, following the careful exposition in [GhS], that her result holds for a wider class of pseudogroups.

3.7. Definition ([Gr 1 1 ). Let $\Gamma$ be a pseudogroup of orientation preserving homeomorphisms between open subsets of $\mathbf{R}$. We say that $\Gamma$ is germ-connected to the identity if, given germs in $\Gamma, g: a \rightarrow$ $c, h: b \rightarrow d$ with $a<b, c<d$, there is an $s \in \Gamma$, whose connected domain contains both $a$ and $b$, such that $\left.s\right|_{a} \equiv g,\left.s\right|_{b} \equiv h$.

3.8. Remark. Evidently, $\Gamma_{H, S}$ is germ-connected to the identity if and only if $H=N$ and $(H, S)$ is flexible.

Let $\Gamma$ be a pseudogroup of orientation preserving homeomorphisms on $\mathbf{R}$. A $\Gamma$-circle is a manifold homeomorphic to $S^{1}$, but with charts 
and gluing data from $\Gamma$; the $\Gamma$-structures $S_{h}^{1}$ constructed above are examples. Not every $\Gamma$-structure, in general, is homotopic to a $\Gamma$ circle. However, if $\Gamma$ is germ-connected to the identity, then any two $\Gamma$-circles are $\Gamma$-homeomorphic (in the obvious sense). Thus, the group $\Gamma\left(S^{1}\right)$ of $\Gamma$-homeomorphisms of a $\Gamma$-circle is well defined. The careful argument in ([GhS], §2) proves:

3.9. Proposition ([GhS], 2.11). Suppose that $\Gamma$ is a pseudogroup of homeomorphisms between open subsets of $\mathbf{R}$, and that $\Gamma$ is germconnected to the identity. There is a map $B \Gamma\left(S^{1}\right) \rightarrow L B \Gamma$ inducing isomorphism on homology, and pulling the Euler class of $L B \Gamma$ back to that of the group $\Gamma\left(S^{1}\right)$.

We conclude with the proof of 1.6.

Proof of 1.6. Since the manifold class of $S_{p, q}^{1}$ is null, the group $\Gamma_{H, S}(p, q)$ exists and is a subgroup of $\Gamma_{H, S}(p, q)$. It suffices to show that the Euler class of $\Gamma_{N, S}(p, q)$ is nonzero and not divisible. Since $(H, S)$ is flexible, $\Gamma_{N, S}$ is germ connected to the identity by 3.8 and we are done, by 3.9 .

\section{REFERENCES}

[Br] Ken Brown, Finiteness properties of groups, J. Pure Appl. Algebra, 44 (1987), 45-75.

[BSq] M. Brin and S. Squier, Groups of piecewise linear homeomorphisms of the real line, Invent. Math., 79 (1985), 485-498.

[Gh1] Etienne Ghys, Groupes d'homeomorphismes du cercle et cohomologie bornée, Contemp. Math., 58 III (1987), 81-106.

[Gh2] —-, Classe d'Euler et minimal exceptionnel, Topology, 26 (1987), 93-105.

[Gh3] _- Sur l'invariance topologique de la classe de Godbillon-Vey, Ann. Inst. Fourier, Grenoble, 37 (1987), 59-76.

[GhS] Etienne Ghys and Vlad Sergiescu, Sur un groupe remarquable de diffeomorphismes du cercle, Comm. Math. Helv., 62 (1987), 185-239.

[Gr1] Peter Greenberg, Pseudogroups from group actions, Amer. J. Math., 109 (1987), 893-906.

[Gr2] _ Pseudogroups of $C^{1}$ piecewise projective homeomorphisms, Pacific J. Math., 129 (1987), 67-75.

[Ha] André Haefliger, Homotopy and Integrability, Springer L. N., 179 (1971), 133-163.

[McD] Dusa McDuff, Foliations and Monoids of Embeddings, in Geometric Topology; ed. Cantrell, Academic Press (1979), 429-444.

[Mi] John Milnor, On the existence of a connection with curvature zero, Comm. Math. Helv., 32 (1957), 215-223. 
[Sh] Goro Shimura, Introduction to the Arithmetic Theory of Automorphic Functions, Princeton University Press (1971).

[W] John Wood, Bundles with totally disconnected structure group, Comm. Math. Helv., 46 (1971), 257-273.

Received May 30, 1990.

UNIVERSITÉ DE GRENOBLE

38402 St. MARTin D'heres CEDEX

FRANCE 



\section{PACIFIC JOURNAL OF MATHEMATICS EDITORS}

\author{
V. S. VARADARAJAN \\ (Managing Editor) \\ University of California \\ Los Angeles, CA 90024-1555 \\ vsv@math.ucla.edu \\ Herbert Clemens \\ University of Utah \\ Salt Lake City, UT 84112 \\ clemens@math.utah.edu \\ F. Michael Christ \\ University of California \\ Los Angeles, CA 90024-1555 \\ christ@math.ucla.edu \\ THOMAs ENRIGHT \\ University of California, San Diego \\ La Jolla, CA 92093 \\ tenright@ucsd.edu
}

\author{
Nicholas ERCOLANI \\ University of Arizona \\ Tucson, AZ 85721 \\ ercolani@math.arizona.edu \\ R. FINN \\ Stanford University \\ Stanford, CA 94305 \\ finn@gauss.stanford.edu \\ VAUGHAN F. R. Jones \\ University of California \\ Berkeley, CA 94720 \\ vfr@math.berkeley.edu \\ Steven KerckhofF \\ Stanford University \\ Stanford, CA 94305 \\ spk@gauss.stanford.edu
}

\author{
C. C. MOORE \\ University of California \\ Berkeley, CA 94720
}

Martin ScharlemanN

University of California

Santa Barbara, CA 93106

mgscharl@henri.ucsb.edu

Harold Stark

University of California, San Diego La Jolla, CA 92093

\section{R. ARENS \\ ASSOCIATE EDITORS}

\begin{tabular}{lccc} 
R. ARENS & $\begin{array}{c}\text { E. F. BECKENBACH } \\
(1906-1982)\end{array}$ & B. H. NEUMANN & \multicolumn{1}{c}{ F. WOLF } \\
$(1904-1989)$ & K. YoshIDA \\
SUPPORTING & INSTITUTIONS \\
UNIVERSITY OF ARIZONA & UNIVERSITY OF OREGON \\
UNIVERSITY OF BRITISH COLUMBIA & UNIVERSITY OF SOUTHERN CALIFORNIA \\
CALIFORNIA INSTITUTE OF TECHNOLOGY & STANFORD UNIVERSITY \\
UNIVERSITY OF CALIFORNIA & UNIVERSITY OF HAWAII \\
MONTANA STATE UNIVERSITY & UNIVERSITY OF TOKYO \\
UNIVERSITY OF NEVADA, RENO & UNIVERSITY OF UTAH \\
NEW MEXICO STATE UNIVERSITY & WASHINGTON STATE UNIVERSITY \\
OREGON STATE UNIVERSITY & UNIVERSITY OF WASHINGTON \\
\hline
\end{tabular}

The Supporting Institutions listed above contribute to the cost of publication of this Journal, but they are not owners or publishers and have no responsibility for its content or policies.

Mathematical papers intended for publication in the Pacific Journal of Mathematics should be in typed form or offset-reproduced (not dittoed), double spaced with large margins. Please do not use built up fractions in the text of the manuscript. However, you may use them in the displayed equations. Underline Greek letters in red, German in green, and script in blue. The first paragraph must be capable of being used separately as a synopsis of the entire paper. In particular it should contain no bibliographic references. Please propose a heading for the odd numbered pages of less than 35 characters. Manuscripts, in triplicate, may be sent to any one of the editors. Please classify according to the 1991 Mathematics Subject Classification scheme which can be found in the December index volumes of Mathematical Reviews. Supply name and address of author to whom proofs should be sent. All other communications should be addressed to the managing editor, or Elaine Barth, University of California, Los Angeles, California 90024-1555.

There are page-charges associated with articles appearing in the Pacific Journal of Mathematics. These charges are expected to be paid by the author's University, Government Agency or Company. If the author or authors do not have access to such Institutional support these charges are waived. Single authors will receive 50 free reprints; joint authors will receive a total of 100 free reprints. Additional copies may be obtained at cost in multiples of 50 .

The Pacific Journal of Mathematics (ISSN 0030-8730) is published monthly except for July and August. Regular subscription rate: $\$ 190.00$ a year (10 issues). Special rate: $\$ 95.00$ a year to individual members of supporting institutions.

Subscriptions, orders for numbers issued in the last three calendar years, and changes of address should be sent to Pacific Journal of Mathematics, P.O. Box 969, Carmel Valley, CA 93924, U.S.A. Old back numbers obtainable from Kraus Periodicals Co., Route 100, Millwood, NY 10546.

The Pacific Journal of Mathematics at P.O. Box 969, Carmel Valley, CA 93924 (ISSN 0030-8730) is published monthly except for July and August. Second-class postage paid at Carmel Valley, California 93924, and additional mailing offices. Postmaster: send address changes to Pacific Journal of Mathematics, P.O. Box 969, Carmel Valley, CA 93924.

PUBLISHED BY PACIFIC JOURNAL OF MATHEMATICS, A NON-PROFIT CORPORATION Copyright (C) 1992 by Pacific Journal of Mathematics 


\section{PACIFIC JOURNAL OF MATHEMATICS}

Volume $155 \quad$ No. $2 \quad$ October 1992

Invariant subspaces and harmonic conjugation on compact abelian

201 groups

NAKHLE HABIB ASMAR, EARL ROBERT BERKSON and

ThOMAS Alastair GiLlESPIE

Cobcat and singular bordism

A. K. DAS and S. S. KHARE

Poles of Eisenstein series on $\mathrm{SL}_{n}$ induced from maximal parabolics

PAUL FEIT

On the postulation of 0-dimensional subschemes on a smooth quadric

251

SAlvatore GiUfFrida, Renato MagGioni and AlFio

RAGUSA

The Euler class for "piecewise" groups

Peter Abraham GREenberg

$L^{p}$-Fourier transforms on nilpotent Lie groups and solvable Lie groups 295 acting on Siegel domains

JUNKO INOUE

Fuchsian moduli on a Riemann surface-its Poisson structure and

Poincaré-Lefschetz duality

KATSUNORI IWASAKI

Interpolation between $H^{p}$ spaces and noncommutative generalizations. 341 I

\section{GILLES PISIER}

The braid index of generalized cables

ROBERT FONES WILLIAMS and ROBERT FONES WILLIAMS $B M O$ and Hankel operators on Bergman spaces 